\title{
Rappresentazioni planimetriche, vedutistiche e tridimensionali per la fortificazione di due isole del Mediterraneo occidentale: Elba e Palmaria (secolo XIX)
}

Planimetric maps, views and three-dimensional representations for the fortification of two western Mediterranean islands: Elba and Palmaria (nineteenth century)

\author{
Valentina de Santi $^{\text {a }}$, Carlo A. Gemignani ${ }^{\text {b }}$, Anna Guarducci ${ }^{\text {c }}$, Luisa Rossi ${ }^{\text {d }}$ \\ ${ }^{a}$ Università della Svizzera Italiana, Lugano, Italy, valentina.desanti@ hotmail.it

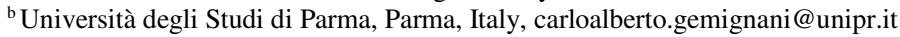 \\ ${ }^{\mathrm{c}}$ Università degli Studi di Siena, Siena, Italy, anna.guarducci@unisi.it \\ ${ }^{\mathrm{d}}$ Università degli Studi di Parma, Parma, Italy, juditta.sp@gmail.com
}

\begin{abstract}
The French expansion and domination in Italy between the Revolutionary Age and the Empire based on a widespread activity of territorial knowledge, which rested in the Corps of Engineers-Geographers and in the Military Genius the main actors. The paper summarizes the results of long research on this activity, carried out in the islands of Elba (Tuscany) and Palmaria (Liguria): two strategic islands in the western Mediterranean. The need to equip the territories dominated by the French with increasingly functional defenses, gave a strong impulse to the renewal of surveying and cartography, with the use of geodetic projections, views and three-dimensional models. Elba example is significant for the complete triangulation of the island connected to the Corsica one (with part of Sardinia and the smaller islands of the Tuscan archipelago). Geographer engineers such as Tranchot, Simonel, Moynet, Puissant worked on these activities and produced some maps and a small model of part of Elba. In the Palmaria example the threedimensional reproduction (plan-relief) was contextual to the work of Genius engineers who produced a vast and organic corpus of maps of various scales, views, sketches and watercolors, suitable to represent the most complete visualization of the landscapes where to insert defensive buildings. The collaboration between French and Italian engineers took advantage of this first experience in designing some batteries. However, it was the post-Napoleonic decades that made Palmaria island a powerful "fortress island" to defend the entrance to the Gulf of La Spezia, where the military arsenal (commissioned by Cavour and built by Domenico Chiodo) arose.
\end{abstract}

Keywords: French geographers engineers, cartography, fortifications, Elba island, Palmaria island.

\section{L'Italia quale laboratorio dell'“utopia cartografica" napoleonica}

L'espansione e la dominazione francese in Italia fra Età rivoluzionaria e Impero si appoggia su una capillare attività conoscitiva del territorio che ha nel corpo degli Ingegneri-geografi e nel corpo del Genio i principali (anche se non unici) attori.
Ingegneri geografi e ingegneri delle fortificazioni, figure delle quali si è analizzato il percorso storico di definizione delle competenze e delle istituzioni di appartenenza, sono tra gli attori di 
una lunga tradizione topografica militare ${ }^{2}$. Benché la separazione tra questi due corpi sia lenta, conflittuale e ancora poco chiara, durante i primi decenni del XIX secolo possiamo rilevare che gli ingegneri geografi descrivono i possibili teatri di guerra al fine di offrire ai generali dell'armata tutte le informazioni necessarie a stabilire i movimenti delle truppe o a offrire la percezione globale dell' assetto territoriale ai fini dell'azione; invece, gli ingegneri del Genio si occupano dell'organizzazione militare del terreno attraverso molteplici interventi urbanistici e infrastrutturali (apparati difensivi, strade, ponti etc.). Il risultato di questa duplice e intrecciata attività è un programma descrittivo multi-scalare e composito che qualche autore ha definito "utopia cartografica" (Pansini, 2002; Quaini, 1987, pp. 4-6): una griglia descrittiva, a maglie sempre più larghe, che si estende progressivamente a tutto il territorio e che, a partire dalla seconda metà del XVIII secolo, subisce un processo di codificazione e di uniformazione crescente. Di tale processo l'epoca napoleonica rappresenta uno dei momenti più significativi: è allora che prende avvio la lenta transizione dall'antica cartografia alla cartografia moderna, dallo spazio discontinuo allo spazio uniforme (Bianchin, 1987, pp. 545-559; Bret, 2008, pp. 81-97; Bousquet-Bressolier, 1995, pp. 93-106). Alla rilevazione cartografica a scale sempre più grandi si accompagna l'analisi qualitativa e quantitativa delle caratteristiche territoriali delle regioni considerate: gli ingegneri militari sono gli autori di un vasto corpus di scritti (memorie e dizionari topografici) che partecipano alla nascita della moderna statistica (Bourguet, 1984, pp. 193-230). A questo bagaglio descrittivo si aggiunge una pluralità di linguaggi figurativi quali vedute e plastici che mostrano la tensione continua verso uno sguardo totalizzante sullo spazio. La descrizione del territorio elaborata sotto il duplice aspetto di forma grafica e forma verbale è finalizzata dunque alla traduzione del coup d'oeil militaire, vale a dire della capacità per il topografo di percepire e descrivere lo spazio secondo le logiche militari: conformazione del territorio, sistemi difensivi, distanze, assi di comunicazione e risorse utili alle truppe (Pansini, 2003, pp. 115134).
In effetti, gli archivi parigini del Service Historique de la Défense (Vincennes) e del Musée des Plans-reliefs (Hôtel des Invalides) conservano un vasto corpus di materiali relativo all'attività degli ingegneri-geografi e degli ingegneri del Genio in territorio italiano. Nei prossimi paragrafi verranno sintetizzati i risultati di lunghe ricerche sulla documentazione prodotta da questi attori nelle isole Elba (Toscana) e Palmaria (Liguria), strategiche nel Mediterraneo occidentale.

\section{Topografi napoleonici all'isola d'Elba}

La maggiore isola dell'arcipelago toscano, rappresentando uno dei siti strategici più importanti dello scacchiere tirrenico, fu sempre al centro degli interessi dei francesi che produssero, specialmente tra il 1790 e il 1814 , una mole incredibile di documenti scritti e cartografici soprattutto sulle strutture fortificate costiere (Gemignani, Guarducci, Rossi, 2017).

In proposito si segnala un gruppo di cartografie (redatte tra il 1791 e i primi anni dell'Ottocento) di particolare valore per la precisione del rilievo trigonometrico in cui l'Elba presenta una configurazione pressoché geometrica. Tali rappresentazioni, veri e propri prodotti di cartografia scientifica, furono eseguiti nell'ambito delle operazioni topografiche e geodetiche dai tecnici dei bureaux francesi che lavorarono al seguito degli eserciti rivoluzionari e napoleonici alla triangolazione della Corsica (alcune figure riportano sul retro: Catasto della Corsica), operazione che, nel 1790-1791, venne estesa dal geodeta Tranchot e dal topografo Louis Moynel all'arcipelago toscano ma non al litorale continentale, e fu poi proseguita, fino alla caduta di Napoleone, da altri ingegneri geografi militari.

Le figure relative all'Elba (Fig. 1), di differente scala, con l'orografia resa a sfumo rafforzato da sommarie curve di livello, oltre che per la configurazione pressoché geometrica, si apprezzano per il dettaglio dei contenuti topografici: i confini giurisdizionali, le risorse (le miniere di Rio, le saline, la tonnara, l'eccellente portuosità, la fertilità dei terreni), gli insediamenti civili, religiosi e militari, con le numerose torri costiere e le due piazzeforti di Portoferraio e Longone (oggi Porto Azzurro). 


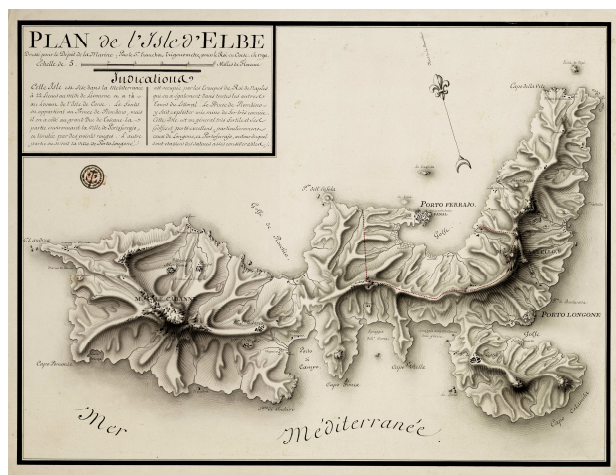

Fig. 1. Plan de l'Isle d'Elbe, Tranchot, 1791; copia conservata a Parigi nella Bibliothèque Nationale de France (GE SH18 PF82 DIV 7 P.8-1 D).

A queste ultime sono dedicate alcune mappe di dettaglio: Plan de Porto Longone en l'Isle d'Elbe e Noveau Plan de Porto-Ferraio (SHD, C.P. M.13.C, cc. 479 e 476). La prima (anonima, di fine Settecento), che potrebbe anche rappresentare un esempio di cartografia di spionaggio funzionale alle esigenze strategiche e militari francesi, in considerazione della legenda in lingua spagnola) rileva con grande esattezza la situazione di fatto di tutti i fabbricati e gli spazi della fortezza (baluardi, chiesa, palazzine del governatore e dei funzionari civili e militari, magazzini, ospedale, etc.), lungo la rada del porto (rivellini, chiese, magazzini, corpi di guardia, etc.) e sulle colline adiacenti (due fonti del Barbarossa e di S. Jacopo), evidenziando pure un progetto di smantellamento di alcuni edifici della "marina e delle alture retrostanti che "disturbavano" le difese. La seconda (Domenico Crivelli, 1800) restituisce la forma urbana del centro marittimo fortificato con il suo immediato entroterra, localizzando (con richiami alfabetici e numerici), tra ampi spazi verdi in parte coltivati, le principali strutture edilizie urbane: le fortificazioni, tra cui il Bastione dei Mulini con ben quattro mulini a vento e le due "Polveriere a prova di bomba", i tre corpi di saline "alla Paesana", "alla Trapanese o di S. Rocco" e "dell'Annunziata", il "Grande Magazzino" del sale "a uso antico di Lazzaretto", "il Forte detto di S. Giovanni Battista demolito e ricostruito di recente dagli inglesi" (poi di nuovo distrutto, come si deduce dall'assenza del colore e dal tratteggio con cui è perimetrata l'area) (Guarducci, 2001; Guarducci, Piccardi, Rombai, 2012).

\section{Dall'Elba alla Palmaria}

Il lavoro dei topografi francesi attivi sul terreno dell'Elba è all'origine della realizzazione di un piccolo plastico della parte occidentale dell'Isola d'Elba conservato presso il parigino Musée des plans-reliefs. Sulla base dei loro rilevamenti il modellino fu costruito a Parigi verso il 1805 dal capitano Pierre-Antoine Clerc (1770-1843) che, pur inquadrato nel corpo del Genio, all'epoca si trovava distaccato presso il Dépôt de la Guerre (Rossi, in corso di pubblicazione) ${ }^{3}$. L'oggetto è interessante in sé ma soprattutto perché chiama in causa il nome di Clerc che, dopo le prime esperienze topografiche sul terreno nei dintorni di Parigi ed alcuni lavori a tavolino, come appunto quello sull'Elba, diventa uno degli autori più importanti nel processo di rinnovamento della topografia. Clerc emerge nella storia della cartografia per aver applicato -per primo in spazi geografici di ragguardevole estensione- il metodo delle curve di livello. A capo della Brigade topographique del Dépôt des Fortifications, creata a sua misura, nel 1809 egli viene inviato dal governo francese nel Golfo della Spezia (Rossi, 2011, in corso di pubblicazione).

La speciale conformazione del Golfo già negli anni precedenti aveva fatto individuare il sito come il più adatto all'impianto di un arsenale (relazioni di Reboul del 1796, di Tagliafichi del marzo 1806, di Lescallier dell'aprile dello stesso anno, di Morlaincourt del 1807); l'entrata della Toscana nell'Impero (ottobre 1807) aveva rappresentato un ulteriore fattore di sicurezza e, con il decreto citato ed altri immediatamente successivi, Napoleone non esitò a dare il via alla grande operazione urbanistica che prevedeva, oltre all'arsenale, diverse opere fortificate, una città nuova e tutte le infrastrutture necessarie (Rossi, 2003, 2008).

Per la città e l'arsenale fu scelta la costa occidentale del golfo, vale a dire il promontorio di Porto Venere e l'isola antistante, appunto la Palmaria che, munita di fortificazioni, ne sarebbe stata la sentinella.

L'aderenza al terreno delle opere previste richiedeva accurate operazioni di rilevamento e, come fu ordinato, la costruzione di un plastico a grande 
scala (1:1000). Planimetrie e plan-relief, restituendo il paesaggio in tutte le sue dimensioni e nei particolari, avrebbe consentito all'imperatore di giudicare da Parigi la validità dei progetti ${ }^{4}$. Esso veniva inoltre ad arricchire la collezione iniziata da Lugi XIV e dal grande architetto Vauban, collezione che, connessa con la fortificazione delle frontiere di Francia, aveva anche il ruolo di celebrare la gloria del sovrano (Faucherre, Roux, 1993; Warmoes, 2007).

Anche il plastico spezzino veniva ad assumere questi significati. Le operazioni di terreno della brigata topografica di Clerc durarono dalla primavera del 1809 al 1811 . Ne risultarono la grande Carte nivelée par courbes horizontales in $18 \mathrm{fogli}$ in scala 1:1000, la sua riduzione 1:5000 messa al netto a Parigi nel 1812, il grande plastico fabbricato in parte in loco e in parte a Parigi, e una straordinaria mole di disegni preparatori, in pianta e in proiezione verticale, non di rado acquarellati.

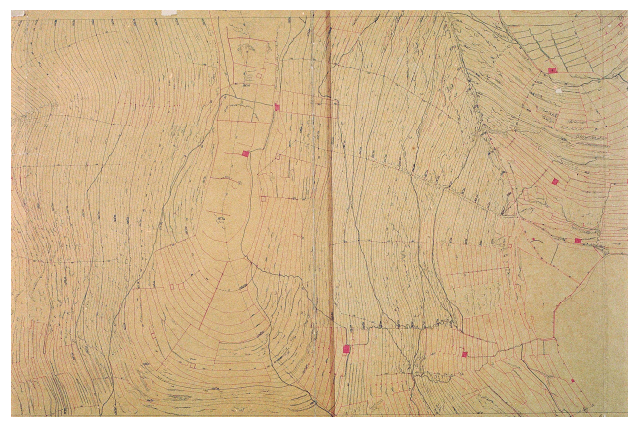

Fig. 2. Il pianoro sommitale della Palmaria rilevata dalla Brigata topografica delle fortificazioni di P.-A. Clerc (particolare), scala originale 1:1000, 1809-1811 (Itituto di Storia e Cultura dell'Arma del Genio/ISCAG, FT 64/A 4033).

Come il resto del territorio considerato, l'isola Palmaria, per la prima volta rappresentata con le curve di livello (Fig. 2), ma anche in tre dimensioni e, inoltre, "pittorescamente" descritta attraverso la visualizzazione di ogni suo elemento paesaggistico -geomorfologia, insediamenti, vegetazione, sistemazioni agrarie etc. (Fig. 3)- costituisce un patrimonio di notevole utilità per gli interventi che verranno progettati nel corso dell'Ottocento (ma preziosi anche gli interventi da effettuarsi alla nostra epoca).

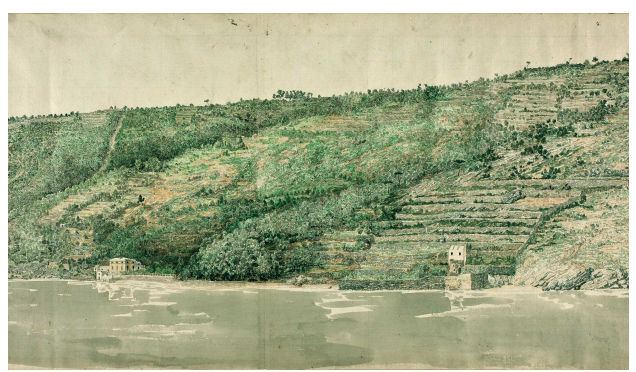

Fig. 3. Brigate topographique, Vue de l'Ile Palmaria en trois feuilles, 1809-1811, particolare (Biblioteca Civica Ubaldo Mazzini, La Spezia, Inv. 317).

\section{L" "isola-fortezza" nelle missioni francesi d'intelligence di epoca postunitaria}

Furono i decenni successivi alla partenza dei topografi comandati da Clerc -soprattutto quelli immediatamente successivi alla proclamazione del Regno d'Italia- il periodo chiave per la trasformazione materiale di un territorio dalla forte impronta rurale ${ }^{5}$ nell' "isola fortezza" di cui ancora oggi scorgiamo l'impronta. Già dai progetti eseguiti -ancora sotto il dominio francese- sotto la supervisione del Comitato centrale delle fortificazioni ${ }^{6}$, emerge immediatamente il senso del forte interesse strategico riservato dai militari al naturale avamposto del Golfo della Spezia rappresentato dalla Palmaria. Il 22 novembre 1813, nonostante lo stesso anno veda la definitiva rinuncia alla realizzazione dell'arsenale da parte francese, François-Marie Riverieulx (1775-1841) firma una Carte de la partie occidentale du Golfe de la Spezia ${ }^{7}$ che consente oggi di determinare con precisione i siti costituenti la rete difensiva "di base", dalla quale avranno origine i successivi concreti interventi compiuti da parte sabauda sull'isola. In essa risultano infatti ancora "in progetto" le batterie costiere della Mariella, quelle della Scola e di "Punta dei Marmi".

Il periodo della dominazione piemontese costituirà una fase di relativa quiete per quanto riguarda gli interventi sulla Palmaria. Sarà a seguito della costruzione dell' arsenale militare marittimo spezzino (voluto da Cavour e realizzato da Domenico Chiodo fra il 1862 e il 1869) che il progetto di rendere l'isola una possente barriera atta a difendere l'ingresso del Golfo diverrà realmente operativa. Le tracce delle installazioni militari 
che in gran parte ancora scorgiamo nel paesaggio attuale sono infatti in gran parte databili alla seconda metà dell'Ottocento, conseguenze materiali della delicata situazione geopolitica che vide per oltre un ventennio Francia e Italia nettamente avverse l'una all'altra (grazie anche all'abile politica disgregatrice operata da Bismark). Siamo oggi a conoscenza di diversi e articolati piani strategici e fitte relazioni ispettive miranti, da parte italiana, a scongiurare una possibile invasione del territorio nazionale compiuta da truppe francesi (Gabriele, 2005; Battaglia, 2013) sia via terra sia dal mare e, dall'altra parte, di dense relazioni spionistiche spesso corredate da materiale cartografico e, in seguito, fotografico. Questa fase di reciproco controllo terminerà soltanto con l'ingresso dell'Italia nella Grande Guerra a fianco delle potenze dell'Intesa (Santi, Gemignani, Rossi, 2018).

Presso il Service Historique de la Défence di Vincennes (SHD Parigi), all'interno della serie denominata in origine Places étrangères, nella sottoserie dedicata all'Italia (1V M 340), è conservato un breve ma denso fascicolo denominato Spezia, che contiene una relazione di 48 pagine più volte aggiornata (1891-1908-1911) riguardante le difese del Golfo, comprese ovviamente quelle poste sulla Palmaria (pp. 29-34). A corredo di questa troviamo una notevole documentazione grafica composta da 20 rilievi topografici, comprendenti anche due carte a scala più piccola, rispettivamente 1:100000 e 1:25000, indicanti tutti i siti di interesse strategico del Levante ligure da Moneglia a Punta Bianca e del Golfo in particolare. Vale la pena di soffermarsi su questo materiale vista la minore notorietà della fonte rispetto ad altre. Il paragrafo più denso (p. 30) è dedicato al Fort Palmaria (poi denominato Forte Cavour) ${ }^{9}$, oggi in stato di totale degrado. Esso venne a occupare la parte sommitale dell'isola indicata in una carta di Giacomo Brusco del 1790 come "Il Piano". Si trattava di un vasto pianoro coltivato a «filagnate di vigna» dal quale digradavano alcuni terrazzamenti a vite e olivo, oltre a pendii coperti di "pini selvatici". Dalla relazione francese l'imponente struttura, la cui costruzione iniziò il 23 marzo 1862, appena la zona fu sottoposta a regime di servitù militare, emerge chiaramente come "un massif de casemates recouvert de terre" (p. 30), cioè di una "copertura a prova di bomba" composta da "terra vegetale" e tegole "alla toscana" per lo scolo dell'acqua piovana, come risulta da altre fonti italiane (Pesaresi, 1999; Luca, Dreini, 2006). Il manufatto era dotato di ben 17 postazioni di artiglieria e torrette telemetriche su ogni bastione. L'anonimo redattore della relazione, forte certamente di informazioni molto precise, ricorda anche la presenza del rivellino posto presso l'ingresso principale; si trattava di un elemento costruttivo interessante, una novità per le fortificazioni del Golfo.

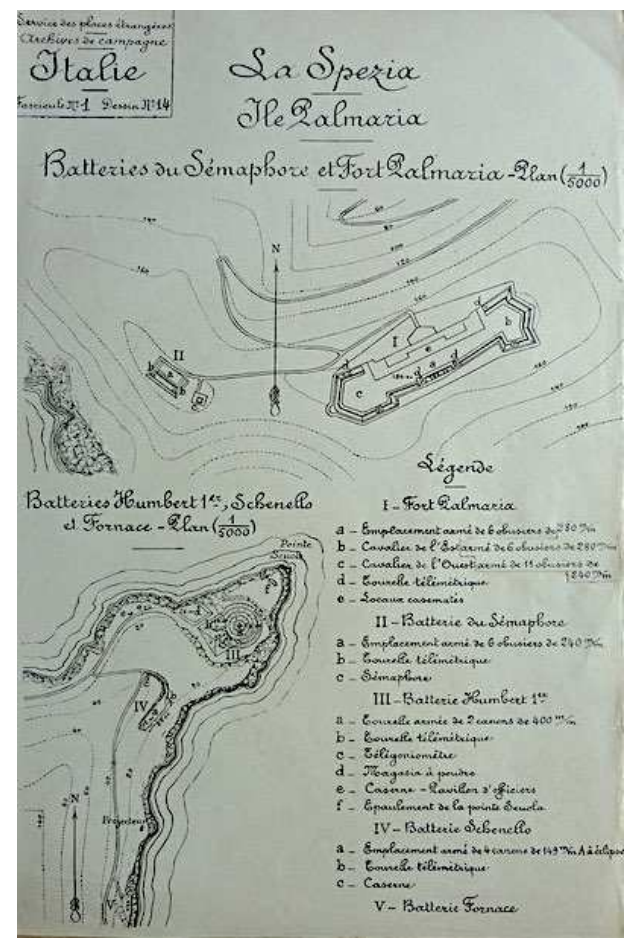

Fig. 4. Forte Palmaria, Batteria del Semaforo e Batteria (o Torre corazzata) Umberto I, piante del disegno originale in scala 1:5000 (SHD, 1V M 340).

Il forte (con la vicina batteria del Semaforo) costituiva il perno centrale di un sistema difensivo che ricalcava perimetro costiero dell'isola comprendente le batterie dello Schenello, della Fornace e del Carlo Alberto (tutte oggi in stato di completo abbandono o addirittura scomparse), e la torre corazzata Umberto I (che è stata, invece, restaurata). Di tutte troviamo una sintetica ma precisa descrizione testuale che si completa con 
le planimetrie ${ }^{10}$ in scala 1:5000 e 1:500 delle due opere principali: il forte sommitale e la batteria Umberto I (Fig. 4). Data la presenza della torre corazzata girevole, edificata tra il 1887 e il 1889 , ospitante i due cannoni Krupp da 400/25 (in grado di scagliare proiettili fino a $5000 \mathrm{~m}$ di distanza), la Torre corazzata Umberto I si delineava come il principale deterrente nei confronti di un attacco navale alle infrastrutture militari del Golfo.

"La tourelle -si legge a p. 33- se manoeuvre au moyen d'accumulateur hydrauliques mis en action en temps ordinaire par une machine à vapeur. On peut suppléer à un arrêt de cette dernière par un dispositif de pompes mues à bras, qui nécessite 40 hommes et un travail de 2 heures pour mettre en charge les accumulateurs et permettre le fonctionnement de la tourelle".

\section{Note}

${ }^{1}$ Gli autori hanno lavorato in stretta collaborazione; in particolare De Santi ha steso il paragrafo 1; Guarducci il par. 2; Rossi il par. 3: Gemignani il par. 4 .

${ }^{2}$ Della vasta bibliografia a disposizione si ricordano alcuni testi ormai classici di riferimento: Berthaut, 1902; Bret, 2008, pp. 81-97; Godlewska, 1999; Konvitz, 1987.

${ }^{3}$ Lo studio del piccolo modello ha consentito di correggere l'errata attribuzione archivistica che lo dava come realizzato nel 1811 in seguito a rilevamenti fatti sul terreno da Clerc e dalla sua brigata topografica (Catalogue de la Galerie des Plans en Relief des places fortes, [1900], Berger-Levrault et C.e Editeurs, Paris/Nancy, p. 25). Le informazioni contenute in questo catalogo erano state riprese anche nei cataloghi successivi.

${ }^{4}$ Diversamente da come sostengono di Grazia e Marinaro, Napoleone non è mai stato alla Spezia. $\mathrm{Va}$ anche segnalato che la carta attribuita da queste autrici a Pierre-Antoine Clerc (Fig. 6, p. 116) non è del topografo francese (di Grazia, Marinaro, 2015, pp. 111-118). Essa fa invece parte della serie delle tavole redatte negli anni Venti dell'Ottocento dai topografi di S. M. il Re di Sardegna i quali, per la rappresentazione del rilievo terrestre, non utilizzano ancora il metodo delle isoipse ma un linguaggio fortemente pittoresco.

5 Di questa vocazione agricola ci dà testimonianza una carta datata 1790 del cartografo genovese Giacomo Brusco (Landi, Rossi, 2013).

${ }^{6}$ A raccogliere l'eredità della brigata topografica, ancora in epoca napoleonica, sarà il sotto-direttore alle fortificazioni della Spezia Francesco Pezzi (1764-1813), già autore di vari progetti di fortificazioni nel Golfo fra il 1809 e il 1812 (Gemignani, 2007, pp. 121-125).

${ }^{7}$ Oggi conservata a Roma presso l'ISCAG (FT, 4030), essa utilizza il rilievo precedentemente realizzato da Clerc.

${ }^{8}$ Fara, 2006, p. 261. Per i numerosi documenti cartografici che la progressiva fortificazione del Golfo ha prodotto si rimanda a Marmori, 1976; Forti, 1992; Rossi, 2007, 2008, e relativa bibliografia.

${ }^{9} \mathrm{~L}$ 'originario progetto del forte era stato tracciato dal generale francese Chasseloup nel giugno del 1810. Nel 1849 questo fu ripreso dal colonnello del Genio sardo Damiano Sauli, nell' ambito degli studi preliminari per il trasferimento dell'Arsenale militare da Genova alla Spezia. Tra il 1857 e il 1859, il capitano del Genio Ernesto Belli elaborò il progetto definitivo che ricalcava quello napoleonico soprattutto per quanto concerne elementi architettonici come i "cavalieri" di ponente e di levante e il fossato continuo.

${ }^{10}$ Sul valore della cartografia per l'inquadramento storico del patrimonio militare del Golfo si veda Gemignani, Cervellini, Rossi, 2017.

\section{Bibliography}

Battaglia, A. (2013). I rapporti italo-francesi e le linee d'invasione transalpina (1859-1882), Nuova Cultura, Roma.

Berthaut, H. (1902). Les ingénieurs géographes 1624-1831: une étude historique, Imprimerie du Service Géographique, Paris. 
Bianchin, A. (1987). "Note in margine all'operare cartografico tra la fine del '700 e l'inizio dell'800", in Cartografia e Istituzioni in età moderna, Atti della Società Ligure di Storia Patria, pp. 545-559.

Bourguet, M.N. (1984). "Dal diverso all'uniforme: le pratiche descrittive nella statistica dipartimentale napoleonica", Quaderni storici, 55, pp. 193-230.

Bousquet-Bressolier, C. (1995). "De la “peinture géométrale” à la carte topographique. Évolution de l'héritage classique au cours du XVIII' siècle", in Bousquet-Bressolier, C., L'œil du cartographe, CTHS, Paris, pp. 93-106.

Bret, P. (2018). "Le moment révolutionnaire: du terrain à la commission topographique de 1802”, in Laboulais, I., dir., Les usages des cartes (XVIII ${ }^{-} X I X^{e}$ siècles). Pour une approche pragmatique des productions cartographiques, Presses universitaires, Strasbourg, pp. 81- 97.

Fara, A. (1983). La Spezia, Laterza, Roma-Bari.

Fara, A. (2006). Napoleone architetto nelle città della guerra in Italia, Olschki, Firenze.

Faucherre, N.; Roux, A. De. (1993). "Les plans en relief de Louis XIV, des outils de travail pour la construction de la frontière", in Corvisier, A., dir., Actes du colloque international sur les plans-reliefs au passé et au présent, SEDES, Paris.

Forti, L.C. (1992). Fortificazioni e ingegneri militari in Liguria (1864-1814), Compagnia dei Librai, Genova.

Gabriele, M. (2005). La frontiera nord-occidentale dall'Unità alla Grande guerra (1861-1915). Piani e studi operativi italiani verso la Francia durante la Triplice Alleanza, Ufficio storico dello Stato maggiore dell'esercito, Roma.

Gemignani, C.A. (2007). "Pezzi, Francesco", in Quaini, M.; Rossi, L., eds., Cartografi in Liguria (secoli XIV-XIX), Brigati, Genova, pp. 121-125.

Gemignani, C.A.; Cervellini, F.; Rossi, L. (2017). "Cartografia e patrimonio militare. Il caso dell'arsenale della Spezia”, in Gemignani, C.A., ed., Officina cartografica. Materiali di studio, Franco Angeli, Milano, pp. 119-136.

Gemignani, C.A.; Guarducci, A.; Rossi, L. (2017). Ricognizioni del Genio e dell'Artiglieria francesi sulle fortificazioni costiere liguri-tirreniche. Interventi e progetti (1810-1813), in Echarri Iribarren V. (Ed), Defensive Architecture of the Mediterranean. XV to XVIII Centuries, Publicacions Universitat d'Alacant, Alacant, vol. 5, pp. 263-270.

Godlewska, A. (1999). Geography Unbound. French Geographic Science from Cassini to Humboldt, The University Chicago Press, Chicago/London.

Grazia, S. Di; Marinaro, L. (2015). "Fortifications and landscape system: geological and geomorphological resilience in the development of the La Spezia Gulf", in FORTMED 2015. Defensive Architecture of the Mediterranean. XV to XVIII centuries, Editorial Universitat Politècnica de València, Valencia, vol. II, pp. 111-118.

Guarducci, A. (2001). "La Toscana nella cartografia militare francese dell'Armée de Terre", L'Universo, LXXXI, 4, pp. 542-560.

Guarducci, A.; Piccardi, M.; Rombai, L. (2012). Atlante della Toscana tirrenica. Cartografia, storia, paesaggi, architetture, Debatte, Livorno.

Konvitz, J.W. (1987). Cartography in France, 1660-1848. Science, Engineering and Statecraft, University of Chicago Press, Chicago-London.

Landi, F.; Rossi, L. (2013). "Se la montagna è un'isola. Riflessioni e ricerche su geografia storica e pianificazione", in Cevasco, R., ed., La natura della montagna. Scritti in ricordo di Giuseppina Poggi, Oltre Edizioni, Sestri Levante.

Luca, G. De; Dreini, A. (2006). “Le fortificazioni nel golfo della Spezia nel XIX sec., in Spagiari P. (a cura di), Castrum, Luna Editore, La Spezia, pp. 157-169.

Pansini, V. (2002). L'œil du topographe et la science de la guerre. Travail scientifique et perception militaire (17601820), Thèse de doctorat EHESS.

Pansini, V. (2003). "Pratique de la description militaire. L'exemple des topographes de l'Armée française (1760-1820) ", in Pratiques de la description, EHESS, Paris, pp. 115-134.

Pesaresi, P. (1999). "Opere fortificate alla Palmaria", in Bosco, G., ed., Progetti integrati per le antiche fortificazioni costiere, Agorà Edizioni, La Spezia, pp. 44-49.

Quaini, M. (1987). "L’utopia cartografica degli ingegneri-geografi nell'età napoleonica”, in Pezzoli, S.; Venturi, S., eds., Una carta del Ferrarese del 1814, Amministrazione Provinciale di Ferrara-Istituto per i beni artistici culturali e naturali della Regione Emilia Romagna, Amilcare Pizzi, Cinisello Balsamo (MI), pp. 4-6. 
Quaini, M.; Rombai, L.; Rossi, L. (1995). La descrizione, la carta, il viaggiatore. Fonti degli archivi parigini per la geografia storica e la storia della cartografia italiana, Università degli Studi di Firenze - Istituto Interfacoltà di Geografia.

Rossi, L. (2007). "Alla periferia dell' Impero: operazioni topografiche francesi nel Golfo della Spezia (1812)", in Casti, E., ed., Cartografia e progettazione territoriale. Dalle carte coloniali alle carte di piano, UTET, Torino, pp. 132.

Rossi, L. (2008). Napoleone e il golfo della Spezia. Topografi francesi in Liguria tra il 1809 e il 1811, Silvana editoriale, Cinisello Balsamo (MI).

Rossi, L. (2011). "La brigade topographique et ses archives", in Salat, N. ; Pénicaut, E., dir., Le Dépôt des fortifications et ses archives 1660-1940, Ministère de la Défense/Archives et Culture, Paris, pp. 97-113.

Rossi, L. (2018). "Pierre-Antoine Clerc et la Brigade topographique du Dépôt des fortifications: premières réalisations des courbes de niveau", Cartes \& Géomatique CFC Revue du Comité Français de Cartographie, 238, pp. 25-38.

Rossi, L. (in corso di pubblicazione). La mesure du paysage, Paris.

Santi, V. De; Gemignani, C.A.; Rossi, L. (2018) "Intorno al 1914-1918. Le frontiere italiane viste dalla Francia”, in Masetti, C., ed., Per un Atlante della Grande Guerra, Labgeo Caraci, Roma.

Warmoes, I. (2007). "Un instrument de cartographie particulier: la collection des plans en relief de Louis XIV”, in D’Orgeix, E.; Sanger, V.; Virol, M.; Warmoes, I., Vauban, la pierre et la plume, Éditions du patrimoine, Paris/Luxemburg, pp. 93-104. 